\title{
Phonon spectral function for an interacting electron-phonon system
}

\author{
J.E. Han and O. Gunnarsson \\ Max-Planck-Institut für Festkörperforschung, D-70506 Stuttgart, Germany
}

(September 14, 2018)

\begin{abstract}
Using exact diagonalzation techniques, we study a model of interacting electrons and phonons. The spectral width of the phonons is found to be reduced as the Coulomb interaction $U$ is increased. For a system with two modes per site, we find a transfer of coupling strength from the upper to the lower mode. This transfer is reduced as $U$ is increased. These results give a qualitative explanation of differences between Raman and photoemission estimates of the electron-phonon coupling constants for $\mathrm{A}_{3} \mathrm{C}_{60}(\mathrm{~A}=\mathrm{K}, \mathrm{Rb})$.
\end{abstract}

In a metallic system a phonon can decay into electronhole pair excitations. This decay contributes to the width of the phonon. It was pointed out by Allen that this additional broadening can be used to estimate the electron-phonon coupling. 6 The width can be measured in neutron scattering or, for the orientationally disordered fullerenes, in Raman scattering. electron-phonon coupling is deduced by assuming noninteracting electrons. 1 The method is, however, often applied to systems with strong correlation due to the Coulomb interaction, such as the alkali-doped fullerenes. In the alkali-doped fullerenes the electron-phonon interaction plays an important role, and accurate estimates of the coupling strength are essential. Almost all experimental estimates for these systems are based on Allen's formula, and the accuracy of this formula is therefore crucial.

In strongly correlated systems the hopping is reduced and the excitation of electron-hole pairs may be more difficult. For instance if the correlation is so strong that the system has a metal-insulator transition, the decay into electron-hole pair excitations is completely suppressed. One aim of this paper is therefore to study how the estimate of the electron-phonon coupling is influenced if the electron-electron interaction is taken into account.

In metals a phonon can decay into a (virtual) electronhole pair excitations which can then decay into a different phonon. In this way there is a coupling between different phonon modes of the same symmetry, leading to new modes which are linear combinations of the old ones. These new modes can have quite different coupling strengths than the old modes. A second aim of this paper is to study how the coupling strength is transferred between the modes due to the coupling via electron-hole pair excitations.

The electron-phonon coupling has been studied extensively for the alkali-doped fullerenes. In particular there have been a study based on neutron scattering, 6 and several studies based on Raman scattering 5 . The high resolution studies of Winter and Kuzmany show a very strong coupling to a few of the low-lying modes, but almost no coupling to the high-lying modes. An alternative approach is based on photoemission from free negatively charged $\mathrm{C}_{60}^{-}$molecules. By studying the weight of vibration satellites, it is possible to deduce the electron-phonon coupling. This results in rather different electron-phonon coupling constants. Although the main coupling was to the low-lying modes, there was also a substantial coupling to the two highest $\mathrm{H}_{g}$ modes. The total coupling strength was also larger than deduced from Raman scattering.

In this paper we study a simple model with electronphonon and electron-electron interactions. We consider a finite cluster with a nondegenerate electronic level and a nondegenerate phonon on each site. This model is solved by using exact diagonalization. We find that the Coulomb interaction reduces the phonon width, and that the use of Allen's formulal therefore leads to an underestimate of the electron-phonon coupling constants in Raman scattering experiments. Furthermore we find that due to the indirect interaction of different phonon modes via electron-hole pair excitations in metallic systems, there is a transfer of coupling strength to the low-lying modes which is not present for a free molecule. Since the Raman measurements of the electron-phonon coupling are performed for a solid, but the photoemission estimate is for a free molecule, the weight transfer is present in the Raman but not in the photoemission estimate. These observations are consistent with differences between the coupling constants deduced from Raman scattering and photoemission.

We consider a model with $N_{\text {mode }}$ nondegenerate phonons per site and with electrons without orbital degeneracy. The Hamiltonian is

$$
\begin{aligned}
H & =\sum_{i \nu} \omega_{\nu} b_{i \nu}^{\dagger} b_{i \nu}+\sum_{i \sigma}\left[\varepsilon_{0}+\sum_{\nu} g_{\nu}\left(b_{i \nu}+b_{i \nu}^{\dagger}\right)\right] n_{i \sigma} \\
& +U \sum_{i} n_{i \uparrow} n_{i \downarrow}+\sum_{i j} t_{i j} c_{i \sigma}^{\dagger} c_{j \sigma}
\end{aligned}
$$

where $i$ labels the $N_{\text {site }}$ sites, $c_{i \sigma}$ and $b_{i \nu}$ annihilate an electron with spin $\sigma$ and a phonon with the label $\nu$, respectively, on site $i$ and $n_{i \sigma}=c_{i \sigma}^{\dagger} c_{i \sigma}$ is an occupation number operator. The energy of the phonon $\nu$ is $\omega_{\nu}$ and its coupling to the electrons is is $g_{\nu}$. The corresponding dimensionless electron-phonon coupling is given by $\lambda_{\nu}=2 g_{\nu}^{2} N(0) / \omega_{p h}$, where $N(0)$ is the density of states per spin. The energy of the electronic level is $\epsilon_{0}$. Two electrons on the same site have a Coulomb repulsion $U$. The hopping between the sites is described by matrix elements $t_{i j}$. A Hamiltonian like (11) with $t_{i j} \equiv t$ for 
the nearest neighbor hopping has a high symmetry and a correspondingly large degeneracy. Since we use exact diagonalization to solve the model, we have to limit the number of sites to a small number (4-6). The resulting one-particle states are then very sparse in energy. Therefore we lower the symmetry by choosing each $t_{i j}$ randomly within some interval, which leads to a denser energy spectrum. The model is solved and the result is then averaged over different sets of $\left\{t_{i j}\right\}$. The strength of the hopping is measured by the one-particle width $W$ of the electronic band (for $g=0$ and $U=0$ ). In this model we for simplicity consider nondegenerate $\left(\mathrm{A}_{g}\right)$ phonons and electrons. In, for instance, $\mathrm{A}_{3} \mathrm{C}_{60}(\mathrm{~A}=\mathrm{K}$, $\mathrm{Rb}$ ) the phonons are five-fold degenerate $\mathrm{H}_{g}$ phonons and the electron states have a three-fold orbital degeneracy. The new physics which may be introduced by these degeneracies, e.g., the Jahn-Teller effect, is not considered here.

We consider a half-filled system, i.e., $N_{\text {site }}$ electrons. With $N_{\text {site }}=6$ there are then 400 different electronic configurations. To obtain a finite size Hilbert space, we limit the maximum number of phonons per modes to $N_{\text {phon }}$. The number of phonon states is then $\left(N_{\text {phon }}+1\right)^{N_{\text {site }}}$ for the case of one mode per site. For instance, with $N_{\text {site }}=6$ and $N_{\text {phon }}=3$, the total Hilbert space has the dimension $1.6384 \cdot 10^{6}$. Such a problem can be solved using exact diagonalization, i.e., the ground-state is expressed as a linear combination of all possible basis states in the Hilbert space. The lowest eigenfunction of the corresponding Hamiltonian matrix is then found using the Lanczos method. We further calculate the phonon Green's function

$$
D_{i j}^{\nu}(t)=-i\left\langle 0\left|T\left\{\phi_{i \nu}(t) \phi_{j \nu}(0)\right\}\right| 0\right\rangle,
$$

where $|0\rangle$ is the ground state, $\phi_{i \nu}(t)=b_{i \nu}(t)+b_{i \nu}^{\dagger}(t)$ is the phonon field operator in the interaction representation and $T$ is the time-ordering operator. Calculation of the Fourier transform gives $D_{i j}^{\nu}(\omega)$. We then define a spectral function as

$$
A_{i i}^{\nu}(\omega)=\frac{1}{\pi}\left|\operatorname{Im} D_{i i}^{\nu}(\omega)\right|,
$$

and study the average $\rho_{p h}(\omega)=\sum_{\nu i} A_{i i} i^{\nu}(\omega) / N_{\text {site }}$. Due to the finite size of the system, the spectrum is discrete. We therefore introduce a Lorentzian broadening with the FWHM (full width at half maximum) $0.01 \mathrm{eV}$.

Fig. 1 shows the phonon spectral function $A(\omega)$ for different values of $U$. Due to the small size of the system, the width of the spectrum should not necessarily be expected to agree with Allen's formula even for $U=0$. Nevertheless, the result of Allen's formula $\gamma_{\text {Allen }}=0.19$, is comparable to the width found for $U=0$. The figure illustrates how the spectral function becomes narrower with increasing $U$. This is further illustrated by the inset, which shows the width of the spectrum, calculated as the mean square deviation of the spectral function. The figure illustrates that one underestimates the electronphonon coupling if Allen's formula is used to extract the coupling for a system with a finite $U$. For systems like $A_{3} \mathrm{C}_{60}(\mathrm{~A}=\mathrm{K}, \mathrm{Rb})$, where the Coulomb interaction is believed to play an important role, the width of the phonons may then be substantially reduced. The use of Allen's formula would then correspondingly underestimate the electron-phonon coupling.

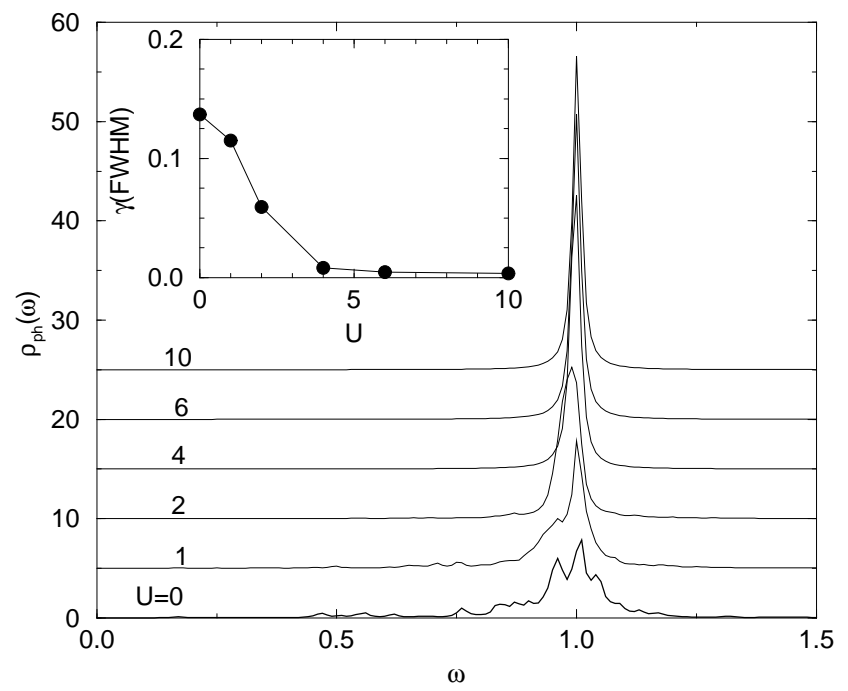

FIG. 1. The phonon spectral function for different values of the Coulomb interaction $U$. The insert shows the width $\gamma$ of the spectral function as a function of $U$. The figure illustrates the narrowing of the spectral function as $U$ is increased. The parameters are $\omega_{1}=1, \lambda=0.073$ and $W=2.5$ and the system has six sites.

We next discuss the case when there are two phonon modes per site, which have the unperturbed energies $\omega_{1}$ and $\omega_{2}$. First we calculate the lowest order phonon selfenergy. This involves evaluating a "bubble" diagram. The self-energy can be written as

$$
\Pi_{\nu, \nu^{\prime}}(\mathbf{q}, \omega) \sim g_{\nu} g_{\nu^{\prime}} f(\omega),
$$

where $f(\omega)$ depends on the precise band structure. We consider contributions to the self-energy which are both diagonal and non-diagonal in the index $\nu$. The nondiagonal contribution corresponds to a phonon $\nu$ decaying into an electron-hole pair followed by this electronhole pair decaying into a phonon $\nu^{\prime}$. The non-interacting phonon Green's function is

$$
D_{\nu, \nu^{\prime}}^{0}(\omega)=2 \omega_{\nu} /\left(\omega^{2}-\omega_{\nu}^{2}\right) \delta_{\nu, \nu^{\prime}} .
$$

The interacting phonon Green's function is then given by

$$
\begin{aligned}
D^{-1}(\omega) & =\left[D^{0}(\omega)\right]^{-1}-\Pi(\omega)= \\
& {\left[\begin{array}{cc}
\frac{\omega^{2}-\omega_{1}^{2}}{2 \omega_{1}}-g_{1}^{2} f(\omega) & -g_{1} g_{2} f(\omega) \\
-g_{1} g_{2} f(\omega) & \frac{\omega^{2}-\omega_{2}^{2}}{2 \omega_{2}}-g_{2}^{2} f(\omega)
\end{array}\right] }
\end{aligned}
$$

The modes of the coupled system are obtained by looking for zeros of the determinant of the matrix in Eq. (6). For 
the lowest mode, the corresponding eigenvector consists of a bonding linear combination of the two unperturbed modes. As a result the coupling to the electrons is increased for these modes, due to constructive interference between the couplings for the two unperturbed modes. In the same way the coupling is reduced for the higher mode. For instance, we can look for the width of the lowest mode in the limit when $\omega_{2} \gg \omega_{1}$ and when the electron-phonon coupling is weak. We then find that the width of the lowest mode is increased by a factor of

$$
\left(1+c \lambda_{2}\right)
$$

and the width of the highest mode is reduced by a factor

$$
\left(1-c \lambda_{2}\left(\frac{\omega_{1}}{\omega 2}\right)^{2}\right)
$$

where $c$ is somewhat larger than unity $(c \sim 3)$ and depends on the shape of the band.

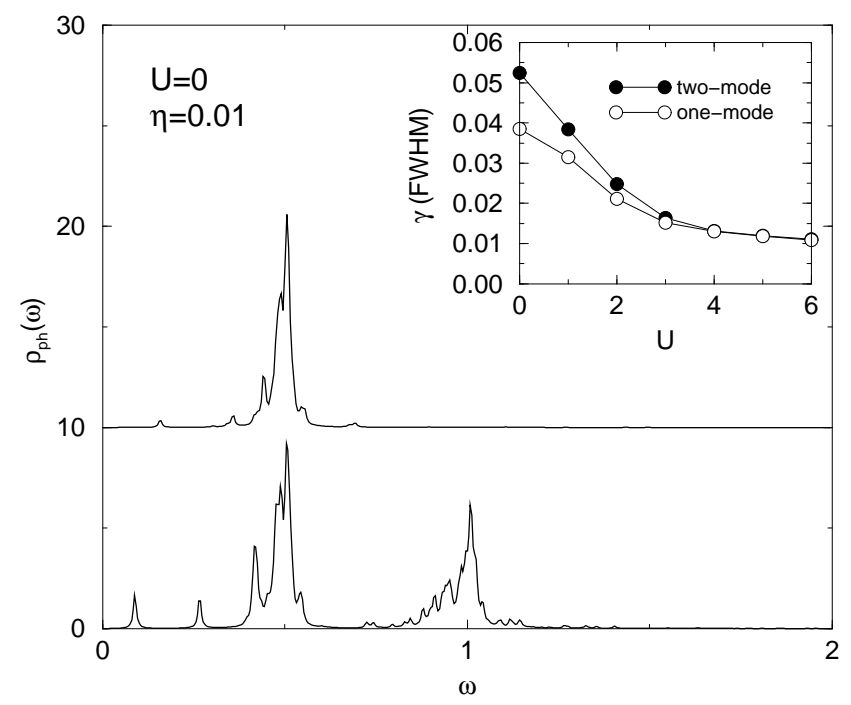

FIG. 2. The phonon spectral function for a system with one phonon mode (upper part) and two phonon modes (lower part) per site for $U=0$. The insert shows the width of the lower mode as a function of $U$ in the cases of one or two modes per site. Comparison of the widths in the two cases, illustrates how the lower mode is broadened for small values of $U$ due to the interaction with the upper mode. The parameters are $W=3.7, \omega_{1}=0.5, \omega_{2}=1, g_{1}=0.3$ and $g_{2}=0.4$ and the system has four sites. All spectra have been given a Lorentzian broadening with the FWHM $=0.1$

The result in Eq. (7) is based on the lowest order phonon self-energy and it neglects the Coulomb repulsion completely. We therefore study the same problem using exact diagonalization. Fig. 2 compares results for systems with one or two modes per site. The discrete spectra have been broadened by a Lorentzian with the $\mathrm{FWHM}=0.01$. The main figure shows results for $U=0$, and it illustrates how the lower mode is broadened when the higher mode is switched on. For the parameters in Fig. $1 \lambda_{1}=0.043$ and $\lambda_{2}=0.085$, and the additional broadening of the lowest mode is of the order of magnitude predicted by Eq. (7). The insert shows the width of the lower mode as a function of $U$. These results were obtained by fitting Lorentzians to the broadened spectra. The width for very large values of $U$ is due to the broadening of the discrete spectrum that we have introduced. As $U$ is increased, the width of the mode is reduced, as discussed above. The figure further illustrates that the transfer of coupling strength is reduced as $U$ is increased. This is expected, since the effects of hopping, and thereby the indirect coupling, is reduced as $U$ is increased.

It would be interesting to repeat these calculations for systems with degenerate phonons, e.g., to include the Jahn-Teller effect. This leads, however, to systems which are so large that they cannot easily be treated using exact diagonalization. Within a Hartree calculation we find a similar transfer of coupling strength to the lower modes also for Jahn-Teller phonons and electrons with orbital degeneracy. The transfer is, however, reduced by the nonspherical parts of the Coulomb interaction, i.e., by the difference between the interaction for equal orbitals and unequal orbitals. This effect may also play a role when we go beyond the Hartree approximation.

Finally, we observe that in theoretical approaches which do not explicitly include the transfer of coupling strength between the modes, it is appropriate to include this transfer by using the corresponding coupling constants. On the other hand, in a treatment where this transfer is explicitly included, the transfer should not be contained in the coupling constants used in the model.

To summarize, we have calculated the phonon spectral functions for systems with interacting electrons and phonons. We find that the Coulomb interaction between the electrons reduces the width of the phonons caused by the phonon decay into electron-hole pairs. As a result, estimates of the electron-phonon coupling based on the phonon width underestimate this coupling unless the Coulomb interaction is taken into account. This is consistent with the observations that weaker couplings have been deduced from Raman measurements than from photoemission (PES) experiments. Furthermore, we find that there is a transfer of coupling strength from the higher modes to the lower modes due to an indirect interaction via electron-hole pairs. This may, at least partly, explain the difference in the distribution of coupling strength between Raman and PES estimates, although it can probably not fully explain the weak coupling to the two highest phonons seen in Raman spectroscopy. In this work we have treated nondegenerate phonons. It would be interesting to extend the work to degenerate, Jahn-Teller phonons, since these are the important phonons in the alkali-doped Fullerenes.

This work has been supported by the Max-PlanckForschungspreis. 
${ }^{1}$ P.B. Allen, Phys. Rev. B 6, 2577 (1972); Solid State Commun. 14, 937 (1974).

2 J.E. Han, O. Gunnarsson, and V. Eyert, Phys. Rev. B 60, 6495 (1999).

${ }^{3}$ O. Gunnarsson, Rev. Mod. Phys. 69, 575 (1997).

${ }^{4}$ K. Prassides, C. Christides, M.J. Rosseinsky, J. Tomkinson, D.W. Murphy, and R.C. Haddon, Europhys. Lett. 19, 629 (1992).

${ }^{5}$ Winter, J. and H. Kuzmany, 1996, Phys. Rev. B 53, 655.
${ }^{6}$ M.G. Mitch, S.J. Chase, and J.S. Lannin, Phys. Rev. Lett. 68, 883 (1992); Phys. Rev. B 46, 3696 (1992).

${ }^{7}$ O. Gunnarsson, H. Handschuh, P.S. Bechthold, B. Kessler, G. Ganteför, and W. Eberhardt, Phys. Rev. Lett. 74, 1875 (1995).

${ }^{8}$ P.W. Stephens, L. Mihaly, P.L. Lee, R.L. Whetten, S.-M. Huang, R. Kaner, F. Diederichs, and K. Holczer, Nature 351, 632 (1991). 